\title{
Super-acceleration in non-minimal derivative coupling model
}

\author{
H. Mohseni Sadjadi, \\ Department of physics, University of Tehran, \\ P.O.B. 14395-547, Tehran, Iran.
}

March 2, 2022

\begin{abstract}
A scalar field model with non-minimal derivative coupling to gravity is considered. It is shown that although in the absence of matter and potential the phantom divide line crossing is forbidden, but for the power law potential and in the presence of matter this crossing is, in principle, possible.
\end{abstract}

\section{Introduction}

Recent astrophysical data indicate that the expansion of our universe is accelerating [1. If the universe is assumed to be filled with perfect fluids, and we adopt the Einstein theory of gravity, nearly $\% 70$ of our universe must be filled by a smooth component with a negative equation of state parameter $w<-1 / 3$, dubbed as dark energy, giving rise to a negative pressure. A natural simple candidate for dark energy, inspired by the scalar field model of inflation, is a dynamical scalar field with a suitable potential, known as the quintessence [2]. Although this model can describe the accelerated expansion, but is unable to explain the phantom divide line crossing which based on some observations may be occurred in recent era [3]. A model consisting of only one scalar field can not describe this crossing, hence another model, comprising a quintessence field and a phantom scalar field (a scalar field with a wrong sign kinetic term [4]), known as quintom model was introduced[5].

By generalizing the models including non-minimal coupling of scalar field to gravity, and by assuming that the coupling terms may also be functions of derivatives of the scalar field, the inflationary cosmological dynamics was discussed in [6]. Late time acceleration of the universe as well as the inflation era may be studied in the framework of such non-minimal coupling models.

*mohsenisad@ut.ac.ir 
When the scalar field is kinetically coupled to the Einstein tensor, the field equation is of the second order and no new degrees of freedom are introduced. The cosmological applications of this model were investigated in [7], and exact solutions were obtained in the absence of matter and potential. In [8], the results of [7] were extended to include the phantom scalar field and also constant potentials. However, finding an exact analytical solution to Friedmann equations in non-minimal derivative coupling model, even for simple kinds of potential, and in the presence of matter is very complicated, and it seems that is not feasible analytically [8]. More generalized models (with additional couplings) and some special cosmological solutions corresponding to power law expansion, both in the late and early time stages of the FLRW universe were also studied in [9]. Extension of non-minimal derivative coupling model to dynamical gravity and aspects of Hawking radiation in this model can be found in [10].

Recently, to reconcile the slowly rolling scalar field (inflaton) with the Higgs boson, the same model in which the unitary bounds are preserved has been used [1].

In this paper by considering a spatially flat FLRW universe and inspired by the model proposed in [11], we consider a slowly rolling scalar field in nonminimal derivative coupling model. We show that besides the acceleration expansion of the universe, this model can describe the super acceleration without using new additional scalar fields. The scheme of the paper is as following:

In the first part of the second section we introduce the model and show that in the absence of the potential and matter, the scalar field can not super accelerate the universe. In the second part by considering a power law potential, and also by taking the matter contribution into the account, we prove that, in principle, solutions describing the phantom divide line crossing may exist in this model. In the third section we conclude the paper.

We use units $\hbar=c=G=1$ throughout the paper.

\section{Phantom divide line crossing in non-minimal deriva- tive coupling model}

We consider a scalar field model with the action

$$
S=\int\left(\frac{R}{16 \pi}-\frac{1}{2} g^{\mu \nu} \partial_{\mu} \varphi \partial_{\nu} \varphi+\frac{k}{2} G^{\mu \nu} \partial_{\mu} \partial_{\nu} \varphi-V(\varphi)\right) \sqrt{-g} d^{4} x+S_{m},
$$

where $G^{\mu \nu}\left(=R^{\mu \nu}-\frac{1}{2} g^{\mu \nu} R\right)$ is the Einstein tensor, and $S_{m}$ is the action of the matter sector. We study the model in a spatially flat FLRW space time equipped with the metric:

$$
d s^{2}=-d t^{2}+a^{2}(t)\left(d x^{2}+d y^{2}+d z^{2}\right)
$$


The Friedmann equations are

$$
H^{2}=\frac{8 \pi}{3}\left(\frac{\dot{\varphi}^{2}}{2}+V(\varphi)+\frac{9 k}{2} H^{2} \dot{\varphi}^{2}\right)+\frac{8 \pi}{3} \rho_{m},
$$

which may be written as

$$
H^{2}=\frac{8 \pi}{3}\left(\frac{\frac{\dot{\varphi}^{2}}{2}+V(\varphi)+\rho_{m}}{1-12 \pi k \dot{\varphi}^{2}}\right),
$$

and

$$
\dot{H}=-4 \pi\left(\frac{\dot{\varphi}^{2}+V(\varphi)+\rho_{m}}{1-12 \pi k \dot{\varphi}^{2}}+\frac{\dot{\varphi}^{2}-V(\varphi)+P_{m}}{1-14 \pi k \dot{\varphi}^{2}}\right)+8 \pi k \frac{H \dot{\varphi} \ddot{\varphi}}{1-4 \pi k \dot{\varphi}^{2}} .
$$

The scalar field equation and the continuity equation for the matter component with energy density $\rho_{m}$, are given by

$$
\begin{aligned}
& \left(1+3 k H^{2}\right) \ddot{\varphi}+\left(3 H\left(1+3 k H^{2}\right)+6 k H \dot{H}\right) \dot{\varphi}+V^{\prime}(\varphi)=0, \\
& \dot{\rho_{m}}+3 H \gamma_{m} \rho_{m}=0 .
\end{aligned}
$$

In terms of the equation of state parameter of the barotropic matter, $w_{m}$, $\gamma_{m}$ is defined by $\gamma_{m}=w_{m}+1$. We have assumed that there is no interaction between the scalar field and the matter component. Friedmann equations can be rewritten as

$$
\begin{aligned}
H^{2} & =\frac{8 \pi}{3}\left(\rho_{d}+\rho_{m}\right) \\
\dot{H} & =-4 \pi\left(P_{d}+\rho_{d}+P_{m}+\rho_{m}\right),
\end{aligned}
$$

where the effective dark component pressure and energy density are defined trough

$$
P_{d}=\frac{\frac{\dot{\varphi}^{2}}{2}-V(\varphi)-2 k H \dot{\varphi} \ddot{\varphi}+4 \pi k \dot{\varphi}^{2} P_{m}}{1-4 \pi k \dot{\varphi}^{2}}
$$

and

$$
\rho_{d}=\frac{\frac{\dot{\varphi}^{2}}{2}+V(\varphi)+12 \pi k \dot{\varphi}^{2} \rho_{m}}{1-12 \pi k \dot{\varphi}^{2}}
$$

respectively. The continuity equation for this effective dark component is

$$
\dot{\rho_{d}}+3 H\left(P_{d}+\rho_{d}\right)=0 .
$$

In contrast to the ordinary scalar field model, the matter contributes in $P_{d}$ as well as in $\rho_{d}$. For $k=0$, where

$$
\dot{H}=-4 \pi\left(\dot{\varphi}^{2}+\gamma_{m} \rho_{m}\right)<0,
$$


we are left with the usual scalar field model which can not cross the phantom divide line characterized by $w=-1\left(w=\frac{P}{\rho}=-1-\frac{2}{3} \frac{\dot{H}}{H^{2}}\right.$ is the equation of state parameter of the universe). But as we will prove, the modification of the kinetic term via non minimal coupling, may enable this crossing (provided that some conditions be satisfied). Let us first investigate the ability of this model to cross $w=-1$ for the most simple case where $V=0$ and the contribution of matter, $\rho_{m}$, is negligible (i.e. a universe dominated by free $\varphi$ component [7]). By defining $X=\left(1+3 k H^{2}\right) \dot{\varphi}$, one can verify that the field equation (6) leads to the autonomous equation

$$
\frac{d X}{d \ln a}=-3 X
$$

with fixed points located at $1+3 k H^{2}=0$ and $\dot{\varphi}=0$. By solving (12) we get

$$
\dot{\varphi}=\frac{C a^{-3}}{1+3 k H^{2}},
$$

where $C$ is a constant. (13) and (4) imply

$$
a^{-6}=\frac{3}{4 \pi C^{2}} \frac{\left(1+3 k H^{2}\right)^{2}}{1+9 k H^{2}} .
$$

By taking time derivative of both sides of (14), we arrive at

$$
\dot{H}=\frac{-3 H^{2}\left(1+3 k H^{2}\right)\left(1+9 k H^{2}\right)}{1+9 k H^{2}+54 k^{2} H^{4}} .
$$

This is the same as what was obtained in [7] (see eq.(22) of [7]) using a different mathematical procedure. This autonomous equation has no real pole and its fixed points are located at $H=0, k H^{2}=-\frac{1}{3}$, and $k H^{2}=-\frac{1}{9}$. It is clear from (14) that $k H^{2}>-\frac{1}{9}$, therefore $\dot{H}<0$ and $\lim _{t \rightarrow \infty} \dot{H}=0$, in agrement with the results of [7], 8]. In this way, $\dot{H}=0$ cannot be crossed and only may be achieved asymptotically. So there is no acceleration to super-acceleration phase transition for the universe in this model.

In the following we investigate if the presence of the matter and the potential can change this situation and super-accelerate the universe. Solving Friedman equations, for a non constant potential even in the absence of matter is a complicated task which based on our knowledge has not yet be done. To get an insight about what happens in the presence of the potential and the matter and to see if the quintessence to phantom phase transition is allowed in this model, let us take the power law potential

$$
V(\varphi)=v \varphi^{n}, \quad v \in \Re,
$$

and try a possible slow roll solution specified by

$$
\ddot{\varphi} \ll 3 H \dot{\varphi},
$$


and [12]

$$
H=h_{0}+h_{1} t^{\alpha}, \alpha(\geq 2) \in N .
$$

If it is found that (18) satisfies the Friedman equations with an even integer $\alpha$ and $h_{1}>0$, then the transition from quintessence phase to phantom phase at $t=0$ is allowed. Note that (18) can be considered as the Taylor series of $H$ around $t=0$, thence $h_{1}$ is proportional to the first non zero derivative of $H$ at $t=0$ and $\alpha$ is the degree of this derivative. Among (4), (5), and (6), two equations are independent. To see if (18) is a solution, we focus on equations (5), and (6).

For slowly varying field (17) the field equation reduces to

$$
\left(3 H\left(1+3 k H^{2}\right)+6 k H \dot{H}\right) \dot{\varphi}+n v \varphi^{n-1}=0 .
$$

By substituting (18) in (19), and in terms of dimensionless quantities $\tilde{h_{1}}:=$ $\frac{h_{1}}{h_{0}^{\alpha+1}}, \tilde{v}:=\frac{v}{h_{0}^{2}}, \tilde{k}:=k h_{0}^{2}$, and $\tau:=h_{0} t$ we obtain

$$
\left(3(1+3 \tilde{k})+\alpha \tilde{k} \tilde{h_{1}} \tau^{\alpha-1}+\mathcal{O}\left(t^{\alpha}\right)\right) \frac{d \varphi}{d \tau}+\tilde{v} \varphi^{n-1}=0 .
$$

The solution of this equation is

$$
\varphi(\tau)=\left(\frac{n(n-2) \tilde{v} \tau \Phi\left(-\frac{6 \alpha \tilde{h_{1}} \tilde{k}}{3(1+3 \tilde{k})} \tau^{\alpha-1}, 1, \frac{1}{\alpha-1}\right)}{3(1+3 \tilde{k})(\alpha-1)}+D\right)^{-\frac{1}{n-2}} .
$$

$\Phi$ is the Lerchphi function defined by

$$
\Phi(x, a, b)=\sum_{n=0}^{\infty} \frac{x^{n}}{(n+b)^{a}},
$$

and $D$ is determined by the value of the scalar field at $t=0, D=\varphi^{2-n}(0)$. In the continue, for the sake of mathematical simplicity we assume $\tilde{k} \gg 1$. As was mentioned in [1] this is the condition for canonical normalization of the field $\varphi$. To see if this solution satisfies the Friedmann equation we proceed as follows:

By expanding $\mathcal{K}:=\dot{\varphi}^{2}$, and $\tilde{V}:=\frac{V}{h_{0}^{2}}$ at $\tau=0$ and after some computation we acquire

$$
\begin{aligned}
& \tilde{\mathcal{K}}:=\frac{\mathcal{K}}{h_{0}^{2}}=\tilde{\mathcal{K}}_{0}+\left(-\frac{8}{3} \tilde{h_{1}} \tilde{\mathcal{K}}_{0}-\frac{18(n-1)}{n} \tilde{k} \frac{\tilde{\mathcal{K}}_{0}^{2}}{\tilde{V}_{0}}\right) \tau+\mathcal{O}\left(\tau^{2}\right) \\
& \tilde{V}=\tilde{V}_{0}-9 \tilde{k} \tilde{\mathcal{K}}_{0} \tau+\mathcal{O}\left(\tau^{2}\right),
\end{aligned}
$$

where the subscript " 0 " denotes the value of the parameter at $t=0$ and

$$
\tilde{\mathcal{K}}_{0}=\frac{n^{2} \varphi(0)^{2(n-1)} \tilde{v}^{2}}{81 \tilde{k}^{4}} .
$$


The matter density also can be expanded at $\tau=0$ to give

$$
\tilde{\rho}_{m}:=\frac{\tilde{\rho}_{m}}{h_{0}^{2}}=\tilde{\rho}_{m 0}-3 \gamma_{m} \tilde{\rho}_{m 0} \tau+\mathcal{O}\left(\tau^{2}\right) .
$$

By substituting (23), (25), and (18) in the Friedmann equation, (5), we obtain :

$$
\begin{aligned}
& \alpha \tilde{h_{1}} \tau^{\alpha-1}+\mathcal{O}\left(\tau^{\alpha}\right)=-4 \pi \mathcal{P} \\
& -\quad 4 \pi\left(\mathcal{A}\left(-\frac{8}{3} \tilde{h}_{1} \tilde{\mathcal{K}}_{0}-\frac{18(n-1)}{n} \tilde{k} \frac{\tilde{\mathcal{K}}_{0}^{2}}{\tilde{V}_{0}}\right)-3 \mathcal{B} \gamma_{m} \tilde{\rho}_{m 0}-9 \mathcal{C} \tilde{k} \tilde{\mathcal{K}}_{0}\right) \tau \\
& +\mathcal{O}\left(\tau^{2}\right)
\end{aligned}
$$

where $\mathcal{P}, \mathcal{A}, \mathcal{B}$ and $\mathcal{C}$ are defined through

$$
\begin{gathered}
\mathcal{P}=\frac{\tilde{\mathcal{K}}_{0}\left(1-8 \pi k \mathcal{K}_{0}+8 \pi k V_{0}\right)+\gamma_{m} \tilde{\rho}_{m 0}-4 \pi(1+3 w) k \tilde{\mathcal{K}}_{0} \rho_{m 0}}{\left(12 \pi k \mathcal{K}_{0}-1\right)\left(4 \pi k \mathcal{K}_{0}-1\right)} \\
\mathcal{A}=\frac{1+4 \pi k(w+3) \rho_{m 0}+8 \pi k V_{0}\left(1-48 \pi^{2} k^{2} \mathcal{K}_{0}^{2}\right)-16 \pi k \mathcal{K}_{0}\left(1-5 \pi k \mathcal{K}_{0}\right)}{\left(12 \pi k \mathcal{K}_{0}-1\right)^{2}\left(4 \pi k \mathcal{K}_{0}-1\right)^{2}} \\
-\frac{96 \pi^{2} k^{2} \rho_{m 0} \mathcal{K}_{0}\left(\gamma_{m}+2 \pi(1+3 w) k \mathcal{K}_{0}\right)}{\left(12 \pi k \mathcal{K}_{0}-1\right)^{2}\left(4 \pi k \mathcal{K}_{0}-1\right)^{2}} \\
\mathcal{B}=\frac{2 w-16 \pi w k \mathcal{K}_{0}\left(3-22 \pi k \mathcal{K}_{0}+48 \pi^{2} k^{2} \mathcal{K}_{0}^{2}\right)}{\left(12 \pi k \mathcal{K}_{0}-1\right)^{2}\left(4 \pi k \mathcal{K}_{0}-1\right)^{2}}
\end{gathered}
$$

and

$$
\mathcal{C}=\frac{8 \pi k \mathcal{K}_{0}\left(1+48 \pi^{2} k^{2} \mathcal{K}_{0}^{2}-16 \pi k \mathcal{K}_{0}\right)}{\left(12 \pi k \mathcal{K}_{0}-1\right)^{2}\left(4 \pi k \mathcal{K}_{0}-1\right)^{2}}
$$

respectively.

Validity of (26) requires: i) $\alpha=2$, ii) $\mathcal{P}=0$, and iii)

$$
\tilde{h_{1}}=-2 \pi\left(\mathcal{A}\left(-\frac{8}{3} \tilde{h}_{1} \tilde{\mathcal{K}}_{0}-\frac{18(n-1)}{n} \tilde{k} \frac{\tilde{\mathcal{K}}_{0}^{2}}{\tilde{V}_{0}}\right)-3 \mathcal{B} \gamma_{m} \tilde{\rho}_{m 0}-9 \mathcal{C} \tilde{k} \tilde{\mathcal{K}}_{0}\right) \text {. }
$$

On the other hand by inserting (21) in (17), we find that the conditions for validity of the approximation (17) in the neighborhood of the transition time $(t=0)$ are

$$
\tilde{h}_{1}=\frac{h_{1}}{h_{0}^{3}} \ll 1, \frac{d^{2} \tilde{V}}{d \varphi^{2}} \ll \tilde{k},
$$

which results in $n(n-1) \tilde{v} \varphi(0)^{n-2} \ll \tilde{k}$ implying $\mathcal{K}_{0} \ll V_{0}$. One can also make use of (4) to obtain $k \mathcal{K}_{0} \ll 1$. With the help of these relations, $\mathcal{P}=0$ reduces to

$$
\gamma_{m} \rho_{m 0} \simeq-8 \pi k \mathcal{K}_{0} V_{0} .
$$


Therefore we must take $k<0$. Besides, from (41) or (7) we find out that the main contribution in $H^{2}$ is coming from the potential: $h_{0}^{2} \simeq \frac{8 \pi}{3} V_{0}$.

By considering $\tilde{k} \tilde{\mathcal{K}}_{0}=k \mathcal{K}_{0}$ and $k V_{0} \tilde{\mathcal{K}}_{0} \ll 1$, from (31) we acquire

$$
\tilde{h}_{1} \simeq 2 \pi\left(72 \pi\left(\frac{3 n-2}{n}\right) k^{2} \mathcal{K}_{0}^{2}+6 \gamma_{m} w_{m} \tilde{\rho}_{m 0}\right) .
$$

Now let us investigate the conditions required for $\tilde{h}_{1}>0$. If $w \neq 0$, then $\tilde{h}_{1} \simeq 12 \pi \gamma_{m} w \tilde{\rho}_{m 0}>0$, and the transition occurs. If $w_{m}=0$, as is expected when $\rho_{m}$ is taken as the cold dark matter, $\tilde{h}_{1}>0$ requires $n>\frac{2}{3}$ or $n<0$. These results show that the transition from quintessence to phantom phase is in principle allowed in this model. At the end let us note that by virtue of (33), the main contribution of the energy density is coming from the scalar field potential, which implies $\rho_{m 0} \ll \rho_{d 0}$. To obtain a same order of magnitude for $\rho_{m}$ and $\rho_{d}$, and to alleviate the coincidence problem one should consider an interaction between these components.

\section{Conclusion}

We investigated whether phantom divide line (defined by $w=-1$, where $w$ is the equation of state parameter of the universe) crossing can be realized in a scalar field model whose the kinetic term is non-minimally coupled to gravity (see (1)). We began our study with a simple model without the matter and the scalar field potential, and showed that super-acceleration is not possible in this situation and $w=-1$ can be only achieved asymptotically (see the discussion after (15)). In the next step we considered the contribution of matter (such as cold dark matter or ordinary matter with positive pressure) and took the power law potential for the scalar field (see (16)). As in this case, obtaining an analytical exact solution to Friedmann equations is not possible, we tried an approximate slowly rolling solution (see(17) and (18)) and showed that the transition from quintessence to phantom phase may be in principle occurred in this model.

\section{References}

[1] S. Perlmutter, et al., Nature (London) 391, 51 (1998); A. G. Riess, et al., Supernova Search Team Collaboration, Astron. J. 116, 1009 (1998); S. Perlmutter, et al., Supernova Cosmology Project Collaboration, Astrophys. J. 517, 565 (1999); S. Nojiri, and S. D. Odintsov, Phys. Lett. B 599, 137 (2004).

[2] E. J. Copeland, A. R. Liddle, and D. Wands, Phys. Rev. D 57, 4686 (1998); R. R. Caldwell, R. Dave, and P. J. Steinhardt, Phys. Rev. Lett. 80, 1582 (1998); V. Faraoni, Class. Quantum Gravity 22, 3235 (2005). 
[3] U. Alam, V. Sahni, T. D. Saini, and A. A. Starobinsky, Mon. Not. R. Astron. Soc. 354, 275 (2004); U. Alam, V. Sahni, and A. A. Starobinsky, J. Cosmol. Astropart. Phys. 0406, 008 (2004); A. Vikman, Phys. Rev. D 71, 023515 (2005); E. A. Lim, I. Sawicki, and A. Vikman, JCAP 1005, 012 (2010); D. Huterer, and A. Cooray, Phys. Rev. D 71 (2005) 023506; S. Nojiri, and S. D. Odintsov, Phys. Rev. D 72, 023003 (2005); H. M. Sadjadi, Eur. Phys. J. C 66, 445 (2010).

[4] R. R. Caldwell, Phys. Lett. B 545, 23 (2002); R. R. Caldwell, M. Kamionkowski, and N. N. Weinberg, Phys. Rev. Lett. 91, 071301 (2003).

[5] B. Feng, X.L. Wang, and X. M. Zhang, Phys. Lett. B 607, 35 (2005).

[6] L. Amendola, Phys. Lett. B 301, 175 (1993).

[7] S. V. Sushkov, Phys. Rev. D 80, 103505 (2009).

[8] E. N. Saridakis, and S. V. Sushkov, Phys. Rev. D 81, 083510 (2010).

[9] L. N. Granda, arXiv:1009.3964v2 [hep-th]; L. N. Granda, and W. Cardona, arXiv:1005.2716v2 [hep-th]; Ch. Gao, arXiv:1002.4035v7.

[10] C. Ding, C. Liu, J. Jing, and S. Chen, JHEP 11, 146 (2010).

[11] C. Germani, and A. Kehagias, Phys. Rev. Lett. 105, 011302 (2010).

[12] S. Nojiri, and S. D. Odintsov, Phys. Lett. B 631, 1 (2005); H. M. Sadjadi, and M. Alimohammadi, Phys. Rev. D 74, 103007 (2006); H. M. Sadjadi, and M. Alimohammadi, Phys. Rev. D 74, 043506 (2006);

H. M. Sadjadi, Phys. Lett. B 687, 114 (2010); M. Alimohammadi, and H. Behnamian, Phys. Rev. D 80, 063008 (2009). 